\title{
Front Matter: Volume 9368
}

, "Front Matter: Volume 9368," Proc. SPIE 9368, Optical Interconnects XV, 936801 (23 April 2015); doi: 10.1117/12.2190480

SPIE. Event: SPIE OPTO, 2015, San Francisco, California, United States 


\title{
PROCEEDINGS OF SPIE
}

\section{Optical Interconnects XV}

\author{
Henning Schröder \\ Ray T. Chen \\ Editors
}

9-11 February 2015

San Francisco, California, United States

Sponsored and Published by

SPIE 
The papers included in this volume were part of the technical conference cited on the cover and title page. Papers were selected and subject to review by the editors and conference program committee. Some conference presentations may not be available for publication. The papers published in these proceedings reflect the work and thoughts of the authors and are published herein as submitted. The publisher is not responsible for the validity of the information or for any outcomes resulting from reliance thereon.

Please use the following format to cite material from this book:

Author(s), "Title of Paper," in Optical Interconnects XV, edited by Henning Schröder, Ray T. Chen, Proceedings of SPIE Vol. 9368 (SPIE, Bellingham, WA, 2015) Article CID Number.

ISSN: 0277-786X

ISBN: 9781628414585

Published by

SPIE

P.O. Box 10, Bellingham, Washington 98227-0010 USA

Telephone +1 3606763290 (Pacific Time) · Fax +1 3606471445

SPIE.org

Copyright $@ 2015$, Society of Photo-Optical Instrumentation Engineers.

Copying of material in this book for internal or personal use, or for the internal or personal use of specific clients, beyond the fair use provisions granted by the U.S. Copyright Law is authorized by SPIE subject to payment of copying fees. The Transactional Reporting Service base fee for this volume is $\$ 18.00$ per article (or portion thereof), which should be paid directly to the Copyright Clearance Center (CCC), 222 Rosewood Drive, Danvers, MA 01923. Payment may also be made electronically through CCC Online at copyright.com. Other copying for republication, resale, advertising or promotion, or any form of systematic or multiple reproduction of any material in this book is prohibited except with permission in writing from the publisher. The CCC fee code is $0277-786 \mathrm{X} / 15 / \$ 18.00$.

Printed in the United States of America.

Publication of record for individual papers is online in the SPIE Digital Library.

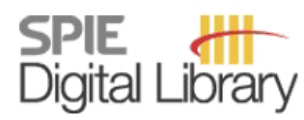

SPIEDigitalLibrary.org

Paper Numbering: Proceedings of SPIE follow an e-First publication model, with papers published first online and then in print. Papers are published as they are submitted and meet publication criteria. A unique citation identifier (CID) number is assigned to each article at the time of the first publication. Utilization of CIDs allows articles to be fully citable as soon as they are published online, and connects the same identifier to all online, print, and electronic versions of the publication. SPIE uses a six-digit CID article numbering system in which:

- The first four digits correspond to the SPIE volume number.

- The last two digits indicate publication order within the volume using a Base 36 numbering

system employing both numerals and letters. These two-number sets start with 00, 01, 02, 03, 04, $05,06,07,08,09,0 A, 0 B \ldots$. 0Z, followed by 10-1Z, 20-2Z, etc.

The CID Number appears on each page of the manuscript. The complete citation is used on the first page, and an abbreviated version on subsequent pages. 


\title{
Contents
}

\author{
vii Authors \\ ix Conference Committee
}

\section{SESSION 1 OPTICAL WAVEGUIDE TECHNOLOGIES}

936802 Multimode/single-mode polymer optical waveguide circuit for high-bandwidth-density on-board interconnects (Invited Paper) [9368-1]

936804 Semi-analytic ray tracing method for time-efficient computing of transmission behavior of PCB level optical interconnects with varying core cross sections [9368-3]

936805 Silicone polymer waveguide bridge for Si to glass optical fibers [9368-5]

\section{SESSION 2 NANOPHOTONICS FOR OPTICAL INTERCONNECTS}

936807 Low-loss mode converter for coupling light into slotted photonic crystal waveguide [9368-7]

936808 High optical coupling efficiency quasi-vertical taper for polymer waveguide devices [9368-8]

936809 High efficiency silicon strip waveguide to plasmonic slot waveguide mode converter [9368-9]

\section{SESSION 3 HYBRID INTEGRATED OPTICAL LINK MODULES}

9368 OA 1060-nm VCSEL-based parallel-optical modules for optical interconnects (Invited Paper) [9368-10]

$9368 \mathrm{OB}$ Multi-wavelength transceiver integration on SOI for high-performance computing system applications [9368-11]

9368 OC A CWDM photoreceiver module for $10 \mathrm{~Gb} / \mathrm{s} \times 4 \mathrm{ch}$ interconnection based on a verticalillumination-type Ge-on-Si photodetectors and a silica-based AWG [9368-12]

\section{SESSION 4 MANUFACTURING TECHNOLOGIES}

$9368 \mathrm{OE} \quad$ Towards high precision manufacturing of 3D optical components using UV-curable hybrid polymers (Invited Paper) [9368-14]

9368 OF Building blocks for actively-aligned micro-optical systems in rapid prototyping and small series production [9368-15] 
$93680 G$ Manufacturability and optical functionality of multimode optical interconnections developed with fast processable and reliable polymer waveguide silicones [9368-16]

$9368 \mathrm{OH} \quad$ Optical coupling structure made by imprinting between single-mode polymer waveguide and embedded VCSEL [9368-17]

\section{SESSION 5 OPTICAL INTERCONNECT DEVICES AND MODULATORS}

9368 OK Broadband energy-efficient optical modulation by hybrid integration of silicon nanophotonics and organic electro-optic polymer [9368-20]

$93680 \mathrm{M}$ Silicon photonics WDM interconnects based on resonant ring modulators and semiconductor mode locked laser (Invited Paper) [9368-22]

\section{SESSION 6 FIBER OPTICS AND MICRO-OPTICS INTEGRATION}

$9368 \mathrm{ON}$ Equilibrium modal power distribution measurement of step-index hard plastic cladding and graded-index silica multimode fibers [9368-23]

936800 Analytical predictive modeling in fiber optics structural analysis (FOSA): review and extension [9368-24]

$9368 \mathrm{OP}$ Theoretical and empirical qualification of a mechanical-optical interface for parallel optics links [9368-25]

$93680 Q \quad$ Polymeric demultiplexer component for wavelength division multiplex communication systems using polymer fibers [9368-26]

9368 OR Planar concave grating with flattened spectral response for wavelength demultiplexing optical interconnection [9368-27]

\section{SESSION 7 OPTICAL INTERCONNECT SYSTEMS}

9368 OT Towards energy-efficient photonic interconnects [9368-29]

9368 OU Electro-optical backplane demonstrator with integrated multimode gradient-index thin glass waveguide panel [9368-30]

9368 OW International standardization of optical circuit board measurement and fabrication procedures [9368-32]

SESSION $8 \quad$ PICS FOR OPTICAL INTERCONNECTS: JOINT SESSION WITH CONFERENCES 9367 AND 9368

9368 OY Design, fabrication, and characterization of nano-imprinted single mode waveguide structures for intra-chip optical communications [9368-34] 
SESSION 9 DEVICE COUPLING APPROACHES FOR SILICON PHOTONICS CHIPS: JOINT SESSION WITH CONFERENCES 9367 AND 9368

$9368 \mathrm{OZ}$ Silicon photonic devices based on SOI/bulk-silicon platforms for chip-level optical interconnects (Invited Paper) [9368-35]

POSTER SESSION

936811 Development and qualification of a mechanical-optical interface for parallel optics links [9368-37]

936812 Multiple-input multiple-output based high density on-chip optical interconnect [9368-38] 


\section{Authors}

Numbers in the index correspond to the last two digits of the six-digit citation identifier (CID) article numbering system used in Proceedings of SPIE. The first four digits reflect the volume number. Base 36 numbering is employed for the last two digits and indicates the order of articles within the volume. Numbers start with 00, 01, 02, 03, 04, 05, 06, 07, 08, 09, OA, OB...0Z, followed by 10-12, 20-2Z, etc.

Aalto, Timo, OB

Alajoki, Teemu, $\mathrm{OH}$

Arndt-Staufenbiel, Norbert, OF

Baghsiahi, Hadi, ON

Baig, Sarfaraz, OR

Boersma, Arjen, $\mathrm{OH}, \mathrm{OY}$

Bosman, Erwin, $\mathrm{OH}$

Böttger, Gunnar, OF

Brusberg, Lars, OU

Chan, Lisa, OG

Chen, Chin-Ta, 09

Chen, Ray T., 07, 08, 09, 0K, 12

Cherchi, Matteo, OB

Chimot, N., OM

Chuang, S., OP, 11

Chung, Chi-jui, 07, OK

Corbett, Brian, $\mathrm{OH}, \mathrm{OY}$

Demir, Yigit, OT

Fernández, F. Aníbal, ON

Fischer, Ulrich H. P., OQ

Gandhi, P., OP

Gruetzner, G., OE

Guo, L. Jay, 08

Han, Joseph, OG

Hardavellas, Nikos, OT

Harjanne, Mikko, OB

Hauck, J., OM

Haupt, Matthias, $0 Q$

Hayashi, Takehiro, ON

Herbst, Christian, OU

Höll, Sebastian, $0 Q$

Hosseini, Amir, 07, 09, 0K, 12

Houbertz, R., OE

Hu, Mike, $0 G$

Huang, Sean, OG

Immonen, Marika, OW

Ishigure, Takaaki, 02

Ishikawa, Y., OA

Islamova, E., OM

Jang, Ki-Seok, OC, OZ

Jen, Alex K.- Y., OK

Jiang, Guomin, OR

Joncic, Mladen, $0 Q$

Joo, Jiho, OC, OZ

Joshi, S., OM

Justice, John, $\mathrm{OH}, \mathrm{OY}$

Kagami, Manabu, ON

Kapulainen, Markku, OB

Karppinen, Mikko, $\mathrm{OH}$, OY
Khan, Umar, $\mathrm{OH}, \mathrm{OY}$

Kim, Gyungock, OC, OZ

Kim, In Gyoo, OC, $\mathrm{OZ}$

Kim, Sanggi, $\mathrm{OZ}$

Kim, Sanghoon, OC, OZ

Kim, Sun Ae, OC, OZ

Kim, Taeyong, OC

Kise, T., OA

Klein, J. J., OE

Kobayashi, Shigeru, ON

Korhonen, $\mathrm{Tia}, \mathrm{OH}, \mathrm{OY}$

Kruse, Kevin L., 05

Kwack, Myung-Joon, $\mathrm{OZ}$

Lang, Klaus-Dieter, OF, OU

Lee, Allen, 0G

Lelarge, F., OM

Li, Qiaochu, 08

Liu, Joe, OG

LU, HUi, OR

Luo, Jingdong, OK

Malacarne, Antonio, OB

Merget, F., OM

Middlebrook, Christopher T., 05

Miller, Allen, OU

Moscoso-Mártir, A., OM

Müller, J., OM

Nagashima, K., OA

Nasu, H., OA

Nekado, Y., OA

Nelson, Robert L., OK

Neumeyr, Christian, OB

Nishimura, N., OA

Oh, Jin Hyuk, OC, OZ

Ortsiefer, Markus, OB

Pan, Zeyu, 07, 08, 09, 0K, 12

Park, Gun Sik, $0 Z$

Park, Hyundai, $\mathrm{OZ}$

Park, Jaegyu, $0 Z$

Park, Jeong Woo, $\mathrm{OZ}$

Persaud, A., OP

Petäjä, Jarno, $\mathrm{OH}$

Pitwon, Richard, OU, OW

Queisser, Marco, OF

Riegel, Nicholas J., 05

Rizky, A. F., OA

Robinson, David, ON

Romero-García, S., OM

Salminen, Noora, $\mathrm{OH}$

Schleunitz, A., OE 
Schoellner, D., OP, 11

Schröder, Henning, OF, OU

Selviah, David R., ON

Sharif Azadeh, S., OM

Shen, B., OM

Shen, Kai, OR

Shen, Po-Kuan, 12

Stübbe, Oliver, 04

Subbaraman, Harish, 07, 08, 0K

Suhir, Ephraim, 00

Swatowski, Brandon W., OG

Tao, Ruichen, ON

Uemura, T., OA

Ugolini, A., OP, 11

Van Steenberge, Geert, $\mathrm{OH}$

Vehmas, Tapani, OB

Vogler, M., OE

Wakjira, J., OP, 11

Wang, Kai, OU, OW

Wang, Michael R., OR

Weber, Daniel, OU

Weidner, W. Ken, OG

Whalley, Simon, OU

Wiegersma, Sjoukje, OY

Witzens, J., OM

Wolf, G., OP, 11

Worrall, Alex, OW

Wu, Jinhua, OW

Xu, Xiaochuan, 09, 12

Yan, Hai, 07, OK

Yan, Hui Juan, OW

Yang, Hui, ON

Yasukawa, Manabu, ON

Ylinen, Sami, OB

Zhang, Cheng, 08

Zhang, Xingyu, 07, 08, 0K

Zhu, Long Xiu, OW

Zou, Yi, 08 


\section{Conference Committee}

Symposium Chairs

David L. Andrews, University of East Anglia

(United Kingdom)

Alexei L. Glebov, OptiGrate Corporation (United States)

Symposium Co-chairs

Jean-Emmanuel Broquin, IMEP-LAHC (France)

Shibin Jiang, AdValue Photonics, Inc. (United States)

Program Track Chair

Yakov Sidorin, Quarles \& Brady LLP (United States)

Conference Chairs

Henning Schröder, Fraunhofer-Institut für Zuverlässigkeit und Mikrointegration (Germany)

Ray T. Chen, The University of Texas at Austin (United States)

Conference Program Committee

Bill Blubaugh, US Conec Ltd. (United States)

Swapnajit Chakravarty, Omega Optics, Inc. (United States)

Patrick B. Chu, Sandia National Laboratories (United States)

Alexei L. Glebov, OptiGrate Corporation (United States)

Michael W. Haney, University of Delaware (United States)

Ruth Houbertz, Multiphoton Optics GmbH (Germany)

Yidong Huang, Tsinghua University (China)

Wei Jiang, Rutgers, The State University of New Jersey (United States)

Mikko Karppinen, VTT Technical Research Centre of Finland (Finland)

Christian Koos, Karlsruher Institut für Technologie (Germany)

Ashok V. Krishnamoorthy, Oracle (United States)

Bert-Jan Offrein, IBM Research - Zürich (Switzerland)

Hyo-Hoon Park, KAIST (Korea, Republic of)

Richard C. Pitwon, Xyratex Technology Ltd. (United Kingdom)

Richard Soref, University of Massachusetts Boston (United States)

Peter Van Daele, Universiteit Gent (Belgium)

Michael R. Wang, University of Miami (United States)

Ian H. White, University of Cambridge (United Kingdom) 


\section{Session Chairs}

1 Optical Waveguide Technologies

Henning Schröder, Fraunhofer-Institut für Zuverlässigkeit und Mikrointegration (Germany)

2 Nanophotonics for Optical Interconnects

Ruth Houbertz, Multiphoton Optics GmbH (Germany)

3 Hybrid Integrated Optical Link Modules

Mikko Karppinen, VTT Technical Research Centre of Finland Ltd. (Finland)

$4 \quad$ Manufacturing Technologies

Peter Van Daele, Universiteit Gent (Belgium)

5 Optical Interconnect Devices and Modulators

Michael R. Wang, University of Miami (United States)

6 Fiber Optics and Micro-Optics Integration

Ian H. White, University of Cambridge (United Kingdom)

7 Optical Interconnect Systems

Ray T. Chen, The University of Texas at Austin (United States)

8 PICs for Optical Interconnects: Joint Session with Conferences 9367 and 9368

Henning Schröder, Fraunhofer-Institut für Zuverlässigkeit und Mikrointegration (Germany)

Graham T. Reed, University of Southampton (United Kingdom)

9 Device Coupling Approaches for Silicon Photonics Chips: Joint Session with Conferences 9367 and 9368

Graham T. Reed, University of Southampton (United Kingdom)

Henning Schröder, Fraunhofer-Institut für Zuverlässigkeit und Mikrointegration (Germany) 